\title{
Auswirkungen des demographischen Wandels in regionaler Differenzierung: Gemeinsamkeiten und Gegensätze ländlich- peripherer Entleerungsregionen in Deutschland - die Beispiele Vorpommern und Westeifel
}

\author{
Anja Reichert-Schick
}

Eingegangen: 27. August 2009 / Angenommen: 3. März 2010 / Online publiziert: 11. Mai 2010

(C) Springer-Verlag 2010

\begin{abstract}
Zusammenfassung Der Beitrag analysiert mit den Regionen Westeifel und Vorpommern zwei periphere ländliche Räume, die vom demographischen Wandel betroffen sind und deutlichen Schrumpfungs- und Alterungsprozessen unterliegen. Aber trotz vieler Gemeinsamkeiten sind gravierende Unterschiede zwischen den Räumen zu konstatieren und der demographische Wandel wird nicht in beiden Regionen gleichermaßen wirksam. Es wird daher der Frage nachgegangen, unter welchen Bedingungen die Brisanz des demographischen Wandels in einer Raumeinheit verschärft oder abgeschwächt wird.
\end{abstract}

Schlüsselwörter Demographischer Wandel · ländliche Räume $\cdot$ Schrumpfung $\cdot$ Eifel $\cdot$ Vorpommern

\section{Impacts of Demographic Change in Regional Differentiation: Similarities and Contradictions Between Rural Areas of Depopulation in Germany- The Example of the Regions West Pomerania and West Eifel}

\begin{abstract}
This article analyses two peripheral rural regions: West Eifel and West Pomerania in Germany. Both are affected by demographic change and are subject to distinct shrinking and ageing processes. There are many similarities between the two regions, but also serious contradictions. The demographic change does not have the same
\end{abstract}

Dr. A. Reichert-Schick $(\triangle)$

Fachbereich VI - Geographie/Geowissenschaften, Wirtschafts- und Sozialgeographie Campus II,

Universität Trier, 54286 Trier, Deutschland

E-Mail: anja.reichert@uni-trier.de effect in both research areas. Hence the article looks into the subject, under which specific conditions the explosiveness of the demographic change is going to be intensified or diminished.

Keywords Demographic change Rural areas Shrinking $\cdot$ Eifel $\cdot$ West Pomerania

\section{Einleitung und Fragestellung}

Die Bevölkerung Deutschlands erfährt durch den demographischen Wandel, der seit den 1970er Jahren eine schleichende, aber grundlegende Umgestaltung der Bevölkerungsstruktur initiiert hat, bedeutsame Veränderungen. Der demographische Wandel weist jedoch einen hohen raum-zeitlichen Differenzierungsgrad auf und die Kommunen befinden sich in unterschiedlichsten Stadien des Wandlungsprozesses. Wachstum und Schrumpfung sind häufig unmittelbar benachbart (vgl. Bucher/Schlömer 2008: 12). Dieser Beitrag analysiert vergleichend zwei schrumpfende ländliche Regionen in Deutschland, wobei bewusst einer Region aus dem äußersten Westen eine weitere aus den neuen Bundesländern gegenübergestellt wird. Es werden die unterschiedlichen Strukturen, Probleme, parallel verlaufende und divergierende Prozesse aufgezeigt, wobei nicht nur der demographische Wandel selbst, sondern notwendigerweise auch seine Verknüpfung mit anderen raumwirksamen Prozessen im Vordergrund steht. Auf Grundlage dieser Analyse schließt der Beitrag mit einer Skizzierung von Entwicklungsperspektiven beider Regionen.

Ländliche Räume werden im aktuellen öffentlichen Diskurs häufig als Problemregionen und Verlierer des demographischen Wandels gesehen. Dies schlägt sich vielfach in reißerischen Schlagzeilen nieder wie „Verlassenes Land, 
verlorenes Land“ (Bölsche 2006) oder „Kleine Dörfer werden einfach von der Landkarte verschwinden“(Peters 2007). Diese provokanten Berichte stellen jedoch lediglich die Aspekte in den Vordergrund, die einen hohen Grad an Publizität besitzen; allerdings muss man feststellen, dass faktisch insbesondere die peripheren ländlichen Räume deutlichen Schrumpfungs- und Alterungsprozessen unterliegen.

Die Kategorie „ländlich“ beschreibt zwar nicht per se einen demographischen Problemraum (vgl. Beetz 2006: 27), aber da ländliche Räume bereits seit vielen Jahrzehnten von der Abwanderung jüngerer Menschen betroffen sind, können sich die Wirkungen des demographischen Wandels dort besonders schnell und intensiv entfalten. Die Folge sind qualitative und quantitative Veränderungen der Bevölkerungs- und Siedlungsstruktur; denn ländliche Abwanderungsregionen haben sich zu überalternden Entleerungsgebieten gewandelt (vgl. Schwedt 1984: 9; Henkel 1978: 1).

Gleichwohl ist zu beobachten, dass der demographische Wandel nicht in allen ländlichen Räumen gleichermaßen wirksam wird. Es stellt sich daher die Frage, unter welchen Bedingungen er eine Region nachhaltig beeinträchtigen kann und unter welchen Bedingungen die Auswirkungen abgemildert werden. In diesem Kontext wird dem Beitrag folgende Hypothese zugrunde gelegt:

Die Brisanz des demographischen Wandels in einer Raumeinheit wird verschärft oder abgeschwächt

1. durch die großräumliche Einbettung einer Region und

2. durch die Ausgangssituation einer Region mit ihren spezifischen naturräumlichen, ökonomischen, gesellschaftlichen und siedlungsgeographischen Besonderheiten und Problemlagen.

\section{Untersuchungsräume und Methoden}

Im März 2007 und im Juni 2008 führte die Verfasserin eine empirische Untersuchung von insgesamt 33 ländlichen Gemeinden in Deutschland durch. ${ }^{1}$ Die beiden Untersuchungsräume liegen sich diametral gegenüber: im Nordosten Deutschlands Vorpommern und im äußersten Westen die Eifel. Beide wurden aufgrund ihres Charakters als Entleerungsregion und Peripherraum mit sehr geringer Bevölkerungsdichte ausgewählt.

Die Entscheidung über die einzubeziehenden Gemeinden erfolgte zunächst mit Hilfe einer Analyse von Sekundärstatistiken. Hierbei wurden bevorzugt Dörfer mit negativer

\footnotetext{
${ }^{1}$ Die Untersuchung erfolgte unter anderem im Rahmen von Forschungspraktika an der Universität Trier, an denen elf bzw. 17 Studierende partizipierten und insbesondere eine umfangreiche Bewohnerbefragung ermöglichten.
}

Bevölkerungsentwicklung berücksichtigt, da in diesen Fällen Auswirkungen des demographischen Wandels im Sinne von Regressionsprozessen zu erwarten sind. Die Anzahl der durch die statistische Analyse in die engere Wahl gezogenen Gemeinden wurde nach Telefoninterviews mit den Bürgermeisterinnen und Bürgermeistern nochmals reduziert.

Die empirische Untersuchung beinhaltete die Befragung von 271 (Vorpommern) bzw. 270 (Westeifel) Einwohnern. Die elf Gemeinden in Vorpommern haben zusammen 3.990 Einwohner, womit die Stichprobe 6,8 \% der Bevölkerung umfasst. Die 22 Gemeinden der Westeifel weisen wesentlich geringere Bevölkerungszahlen auf, wodurch der große Unterschied in der Zahl der Untersuchungsgemeinden bedingt ist. Sie zählen zusammen 3.532 Einwohner. Die Stichprobe umfasst demnach 7,6 \% der in den Dörfern lebenden Bevölkerung. Darüber hinaus wurden umfangreiche qualitative Interviews mit insgesamt 55 Schlüsselpersonen geführt, darunter Bürgermeister, Amtsvorsteher, Landräte, Leiter von Planungsverbänden und Immobilienmakler.

Die Untersuchungsgemeinden befinden sich zum einen mit der Westeifel in einer durch Wald und Grünland geprägten Mittelgebirgsregion, die an Luxemburg und Belgien angrenzt (vgl. Abb. 1). Das dem Untersuchungsraum am nächsten liegende Oberzentrum ist das sich südlich anschließende Trier. Mittelzentren sind die Städte Bitburg und Prüm. Weitere Untersuchungsgemeinden befinden sich im Vorpommerschen Binnenland, das im Südwesten an die Müritz, im Osten an Polen mit der Stadt Stettin und im Norden an die Ostseeküste mit der Insel Usedom grenzt (vgl. Abb. 2). Oberzentren in Vorpommern sind Neubrandenburg und Greifswald, das diese Funktion mit Stralsund teilt. Als Mittelzentren fungieren Demmin, Anklam, Pasewalk, Neustrelitz und Waren an der Müritz sowie Prenzlau im Bundesland Brandenburg.

\section{Vorpommern und Westeifel - eine vergleichende Analyse}

Die vergleichende Analyse der Untersuchungsregionen wird anhand einer kontrastierenden Betrachtung der Gemeinsamkeiten und Unterschiede zwischen der Westeifel und Vorpommern vorgenommen. Auf diese Weise können parallel verlaufende Entwicklungen und divergierende Prozesse abgeleitet werden, um die Ausprägungen und Auswirkungen des demographischen Wandels zu ermitteln.

\subsection{Gemeinsamkeiten}

Zur Betrachtung der Gemeinsamkeiten werden zunächst grundlegende Raumstrukturdaten gegenübergestellt. Im Anschluss werden weitere Analogien anhand der Aspekte 

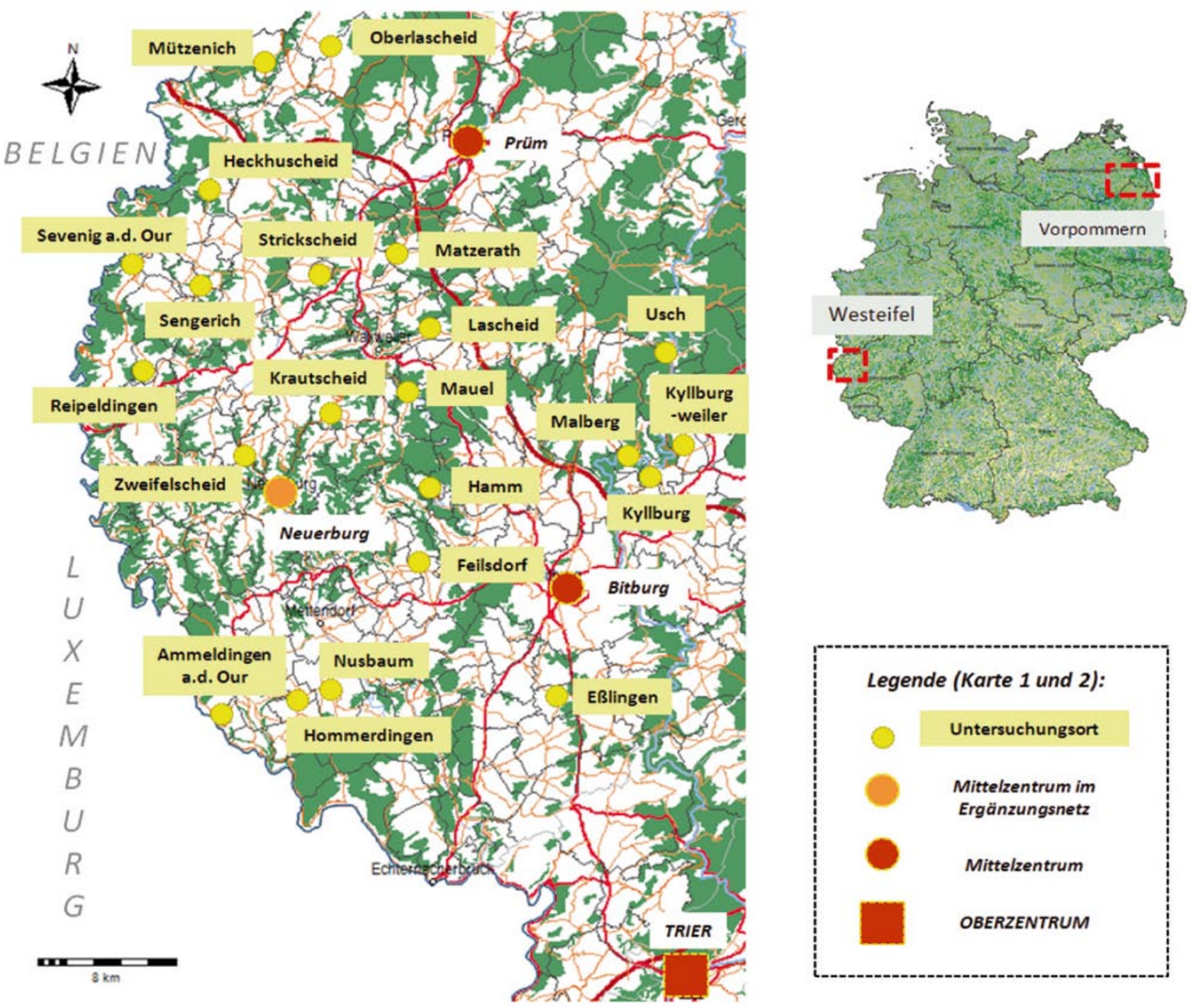

Abb. 1 Untersuchungsgemeinden in der Westeifel

Bevölkerungsentwicklung, Infrastruktur und Umzugsbereitschaft der Bevölkerung herausgearbeitet.

\subsubsection{Strukturvergleich}

Bei einem Strukturvergleich erkennt man, dass die Untersuchungsräume sich vor allem hinsichtlich der klassischen Eigenschaften ländlicher Räume sehr ähnlich sind.

- Ihre Bevölkerungsdichte ist sehr niedrig und bewegt sich in den Landkreisen Vorpommerns zwischen 39 und 56 Einwohnern pro Quadratkilometer. Im Eifelkreis Bitburg-Prüm werden 59 Einwohner pro Quadratkilometer erreicht.

- Das Netz der Zentralen Orte ist sehr weitständig und beide Regionen sind gemäß der Einstufung des Raumordnungsberichtes 2005 als Peripherräume sehr geringer
Dichte zu charakterisieren (vgl. Bundesamt für Bauwesen und Raumordnung 2005: 20). Der Raumordnungsbericht 2000 kennzeichnete die Regionen zusätzlich mit der Eigenschaft (sehr) starker Entwicklungsprobleme (vgl. Bundesamt für Bauwesen und Raumordnung 2000: 65).

- Beide Regionen stellen auf Deutschland bezogen nationale Randräume dar. Bei Vorpommern bezieht sich diese periphere Lage nicht nur auf eine deutschlandweite Betrachtung; denn auch innerhalb des Landes Mecklenburg-Vorpommern, dessen Landeshauptstadt Schwerin sich ganz im Westen befindet, liegt der Untersuchungsraum abgeschieden (vgl. Wichmann 2007: 68).

Darüber hinaus haftet sowohl Vorpommern als auch der Westeifel ein Negativ-Image an. Die Eifel gilt als abgeschiedenes, ärmliches Randgebiet, das aufgrund des rauen 


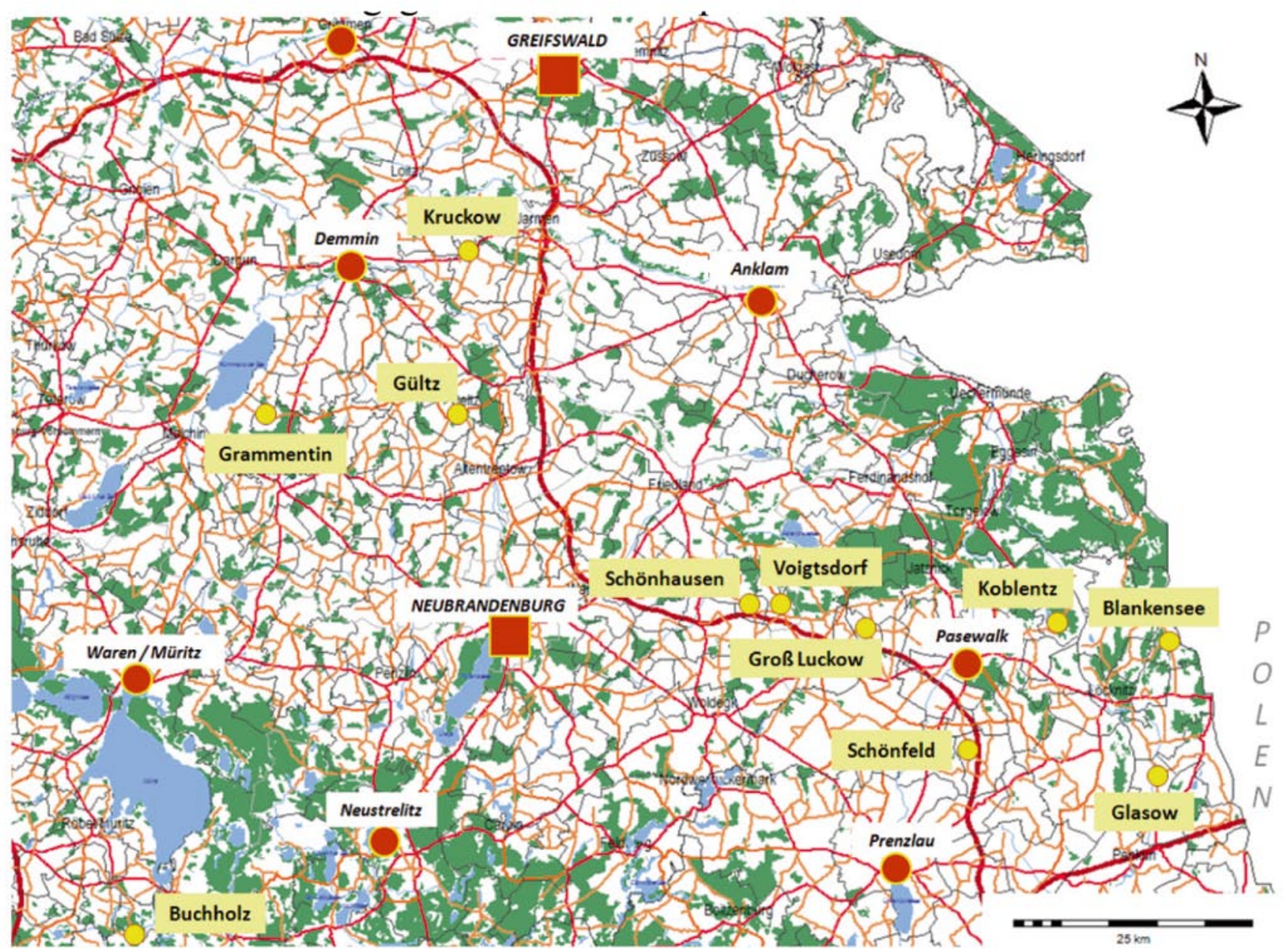

Abb. 2 Untersuchungsgemeinden in Vorpommern

Klimas und der schlechten Böden als „Preußisch Sibirien" tituliert wurde (vgl. Doering-Manteuffel 1995: 62). Vorpommern hingegen wird immer wieder als Problemregion ohne Zukunft bezeichnet, die mittlerweile wieder von Wölfen besiedelt werde (vgl. stellvertretend Peters 2007).

\subsubsection{Bevölkerungsentwicklung}

Eine weitere Gemeinsamkeit stellt die Abwanderung der Bevölkerung dar. Abbildung 3 zeigt, dass die Ausdünnung beider Regionen seit 1971 ungebrochen anhält. In Vorpom-
Abb. 3 Bevölkerungsentwicklung in den Untersuchungsgemeinden der Westeifel und Vorpommerns (gemittelter Wert) zwischen 1971 und $2007(1971=100)$

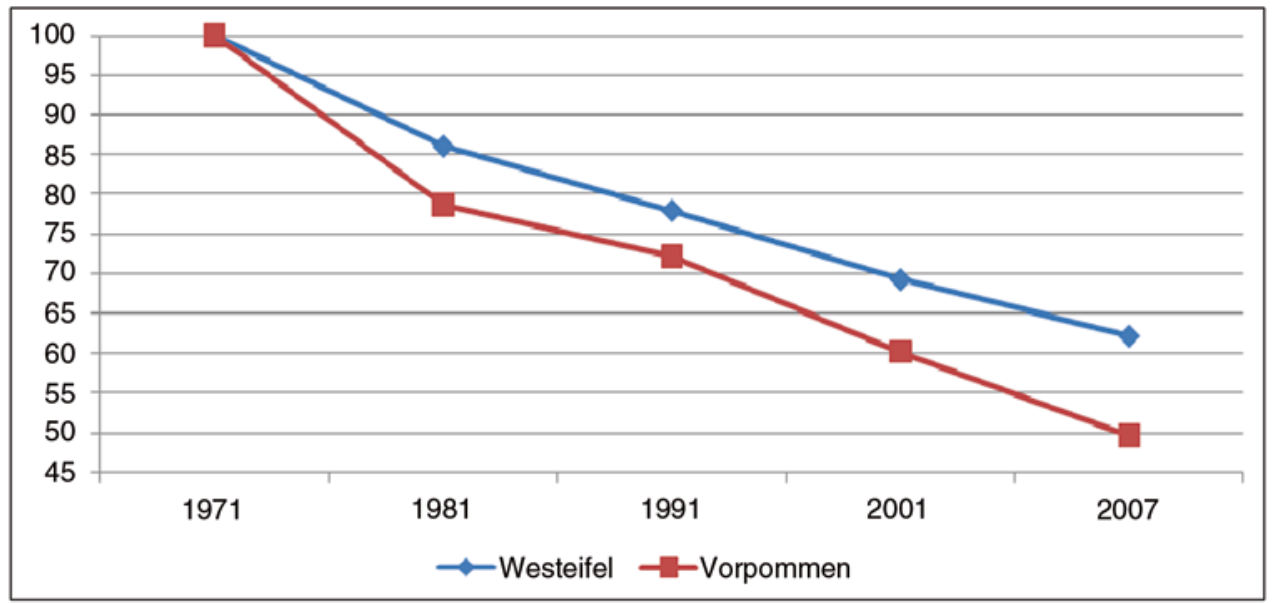



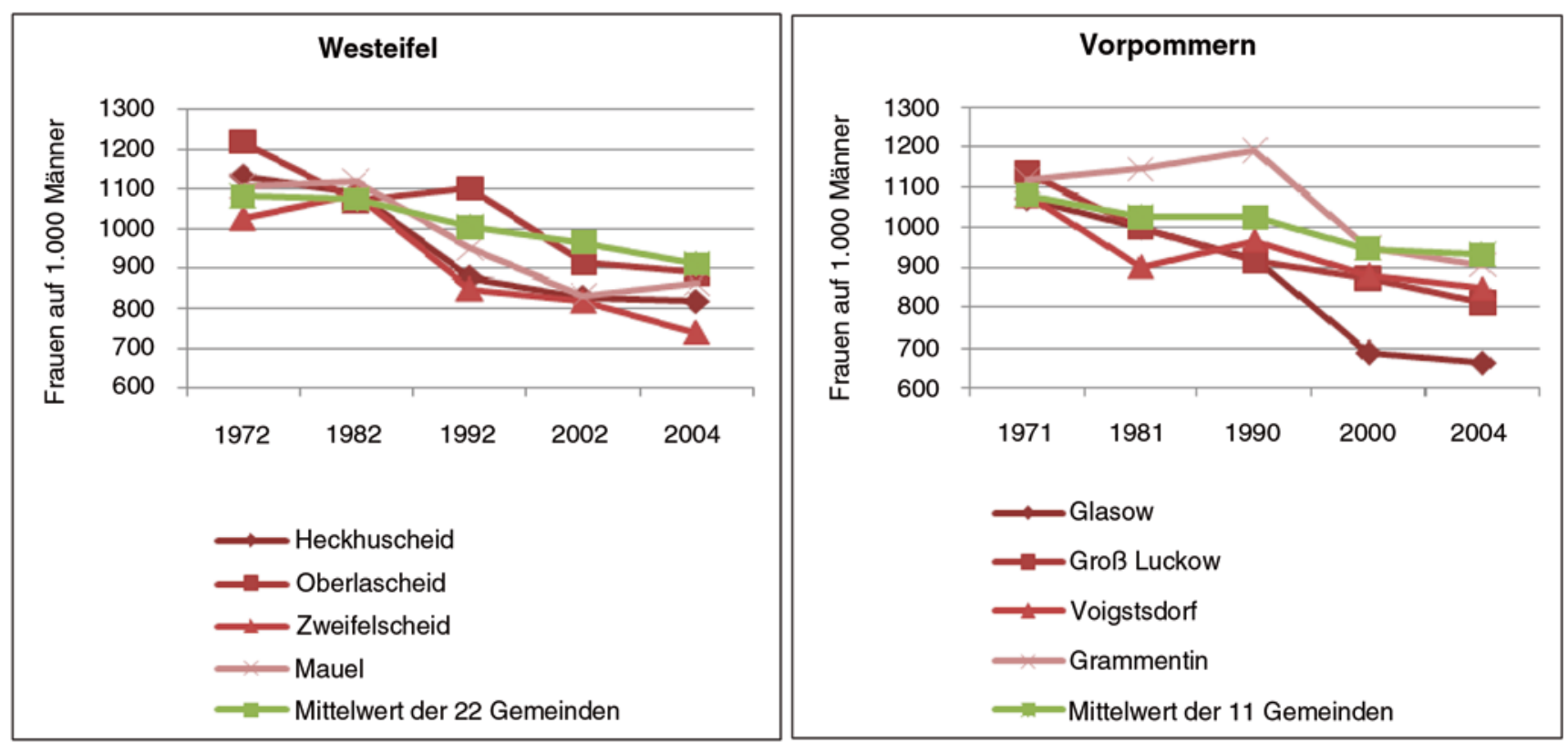

Abb. 4 Geschlechterverhältnis in ausgewählten Untersuchungsgemeinden (im Vergleich zum Mittelwert aller Untersuchungsgemeinden der jeweiligen Untersuchungsregion) für ausgewählte Jahre zwischen 1971 und 2004

mern ist selbst die Wiedervereinigung kaum als Einschnitt wahrnehmbar. Die Untersuchungsgemeinden in Vorpommern haben seither durchschnittlich die Hälfte $(50,4 \%)$ ihrer Bevölkerung verloren; jene in der Westeifel verzeichnen mit minus 37,8 \% etwas geringere Verluste. Die Spannweite des Bevölkerungsverlustes reicht in Vorpommern von 33 (Koblentz) bis $62 \%$ (Voigtsdorf) und in der Westeifel von 14 (Nusbaum) bis $62 \%$ (Hamm). Dabei kann allgemein festgestellt werden, dass kleinere Siedlungen stärker vom Bevölkerungsrückgang betroffen sind.

In beiden Regionen sind die Bevölkerungsverluste in erster Linie auf Wanderungen zurückzuführen. Dabei sind junge Frauen an der Migration überproportional beteiligt, da für sie neben der wenig attraktiven Landwirtschaft kaum alternative Erwerbsmöglichkeiten zur Verfügung stehen und sie zudem aufgrund guter schulischer Abgangsleistungen eher berufliche Positionen in urbanen Räumen annehmen (vgl. Weiß 2006: 483/490). Dieses selektive Wanderungsverhalten hat ein zum Teil deutlich ausgeprägtes Frauendefizit zur Folge (vgl. Abb. 4) und es wurden bereits auffällige Werte von nur noch 600 bis 700 Frauen auf 1.000 Männer erreicht. Der Mittelwert verläuft zwar gemäßigter, aber auch dieser macht die Tendenz sehr deutlich. Durch diese Entwicklung wird die demographische Alterung der Regionen erheblich beschleunigt.

Die Konsequenzen der Abwanderung vorwiegend jüngerer Bevölkerung schlagen sich zudem in demographischen Maßzahlen nieder (vgl. Abb. 5). Der Altenquotient in beiden Regionen liegt deutlich über dem gesamtdeutschen Wert von 31 , wobei in der Westeifel eine ausgeprägtere Alterung festzustellen ist als in Vorpommern. Auch der Jugendquotient zeigt, dass der Anteil von Kindern und Jugendlichen in den Untersuchungsregionen, insbesondere in Vorpommern, weit unter dem deutschen Wert liegt. Die negativen Billeter-Maßzahlen schließlich weisen auf die geringe demographische Entwicklungsmöglichkeit hin. Aus bevölkerungsgeographischer Sicht kann demnach für beide Regionen eine schrumpfende und alternde Gesellschaft konstatiert werden, deren Werte sich in Zukunft vermutlich weiter zu ihrem Nachteil verändern werden.

\subsubsection{Infrastruktur}

Auch hinsichtlich des Infrastrukturabbaus gibt es Gemeinsamkeiten zwischen den Untersuchungsregionen. Die Ausstattung der Dörfer wird zunehmend zugunsten der Zentren eingeschränkt (vgl. Kocks 2004: 24). Jahr für Jahr schließen wichtige Einrichtungen wie Lebensmittelgeschäfte, Bäcker, Postagenturen, Schulen, Kinderbetreuungseinrichtungen und Dorfgaststätten. Auch die Zahl der Arztpraxen sinkt kontinuierlich. Die Dienstleistungen und öffentlichen Einrichtungen werden zunehmend in Zentralen Orten konzentriert, wodurch alltägliche Erledigungen ohne Pkw kaum noch leistbar sind. Dieser Infrastrukturabbau führt für die Betroffenen $\mathrm{zu}$ einem erheblichen organisatorischen und finanziellen Aufwand zur Bewältigung des Alltags.

Abbildung 6 macht deutlich, dass Vorpommern vom Abbau der Infrastruktur noch stärker betroffen ist als die Westeifel. Dies hängt damit zusammen, dass die Dörfer zur DDR-Zeit sehr gut mit Versorgungseinrichtungen ausgestattet waren, was jedoch nach der Wiedervereinigung nicht mehr tragfähig war. Der infrastrukturelle Kahlschlag erfolgte 


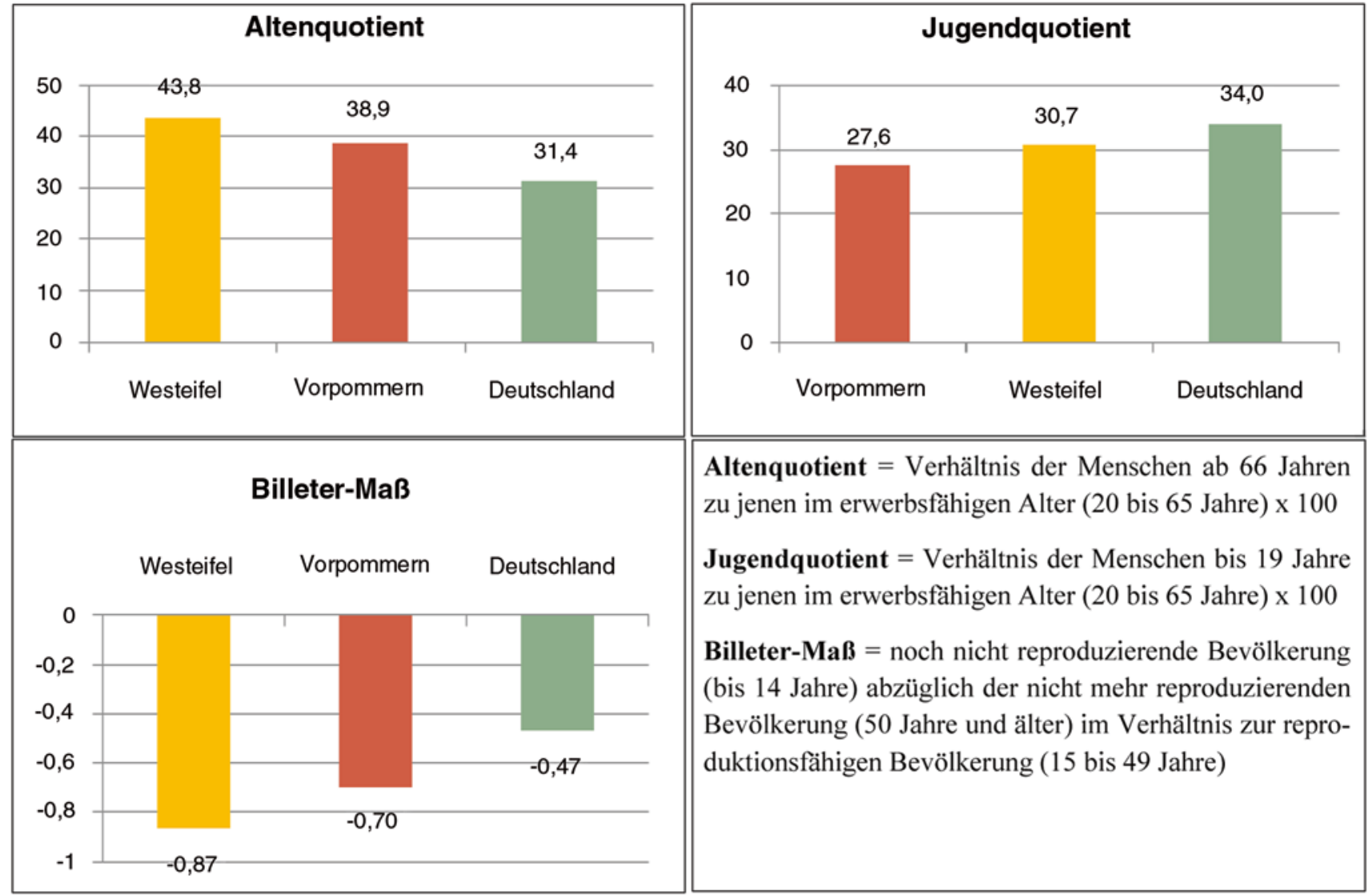

Abb. 5 Demographische Maßzahlen im Vergleich

sehr massiv und schlagartig (vgl. Reichert-Schick 2008: 40-41). Heute besitzen nur noch vier der elf Gemeinden in Vorpommern Versorgungs- oder Dienstleistungseinrichtungen jener Art, welche in dieser empirischen Untersuchung erhoben worden sind (vgl. Abb. 6). So ist beispielsweise in Groß Luckow nach der Schließung der Bäckerei, der Post, der Kindertagesstätte, der Schule und der Arztpraxis noch das Lebensmittelgeschäft erhalten geblieben. In Grammentin ist noch die Kindertagesstätte geöffnet, während Lebensmittelgeschäft, Bäckerei, Metzgerei und Post geschlossen wurden.

Die Dörfer der Westeifel hingegen waren nie in dem Maße ausgestattet wie jene Vorpommerns. Fünf der 22 Untersuchungsgemeinden besaßen sogar nie irgendeine der erhobenen Versorgungs- oder Dienstleistungseinrichtungen, was aber auch auf deren geringe Größe zurückzuführen ist.

Unabhängig von der Anzahl der geschlossenen Einrichtungen, ist der heutige Status Quo für beide Regionen vergleichbar. In den meisten Dörfern besteht gar keine Gelegenheit zur Grundversorgung mehr. Viele Siedlungen wurden in ihren Funktionen weitgehend auf das Wohnen reduziert. Diese Prozesse können letztlich eine weitere Abwanderung initiieren und entziehen den Ortschaften zudem ihre Attraktivität als Standort für Freizeitwohn- und
Altersruhesitze, sofern dieser Aspekt nicht durch andere Vorzüge wie z. B. naturräumliche Werte kompensiert werden kann.

\subsubsection{Umzugsbereitschaft}

Schließlich wurde die Abwanderungsabsicht der Einwohner erfragt (vgl. Abb. 7). In diesem Bereich sind sich die Bewohner der Westeifel und Vorpommerns bemerkenswert einig: Auf einer Skala von 1 bis 8 positionieren sich $66,3 \%$ (Vorpommern) bzw. 70,5\% (Westeifel) bei der 8 , was bedeutet, dass sie ganz sicher nicht umziehen wollen. Nur etwa ein Fünftel der Befragten (Westeifel 18,7 \%; Vorpommern 20,0 \%) bewegt sich im Zustimmungsbereich der Skala zwischen 1 und 4, der einen Umzug in Betracht zieht oder bereits fest intendiert (vgl. Abb. 7).

Dieses Ergebnis wird noch weiter untermauert durch die Aussage von jeweils drei Viertel der Befragten, dass sie sich in ihrer Gemeinde verwurzelt fühlen. Darüber hinaus können sich auch nur wenige vorstellen, den ländlichen Raum als Wohnstandort zu verlassen. Zwar würde rund ein Drittel (31\% Vorpommern, $36 \%$ Westeifel) noch eine Kleinstadt in Betracht ziehen, aber eine Großstadt kommt nur für etwa jeden Siebten in Frage (13\% Vorpommern, $15 \%$ Westei- 


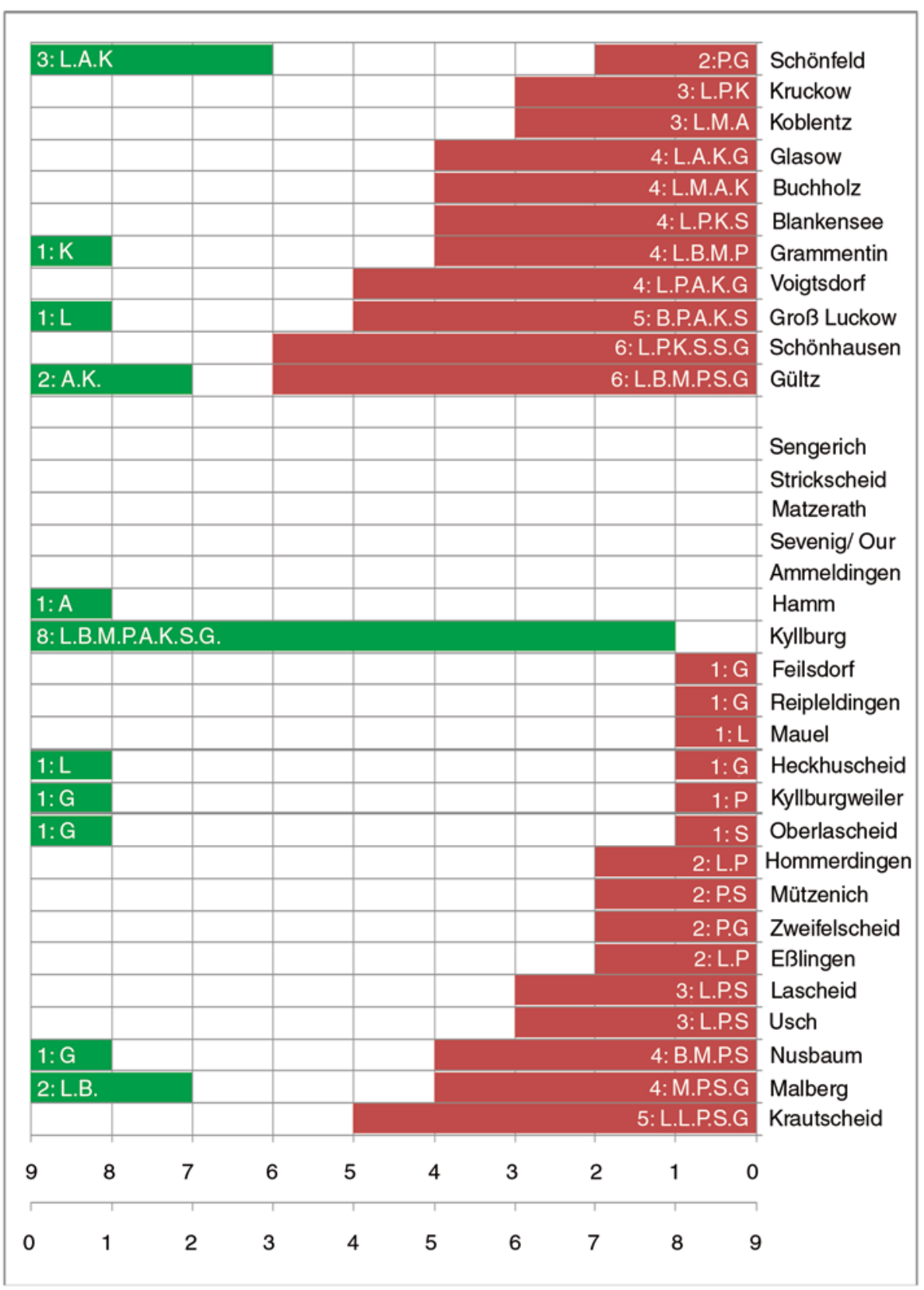

Erhoben wurden:

$\mathrm{L}=$ Lebensmittelgeschäfte

$\mathrm{B}=$ Bäcker

$M=$ Metzger

$\mathrm{P}=$ Post $/$-agenturen

$A=$ Allgemeinarzt

$\mathrm{K}=$ Kinderbetreuung

$\mathrm{S}=$ Schule

$\mathrm{G}=$ Dorfgaststätten

Anzahl der seit 1950 geschlossenen Infrastruktureinrichtungen

Anzahl der noch vorhandenen Infrastruktureinrichtungen

Abb. 6 Geschlossene und vorhandene Infrastruktur in den Untersuchungsgemeinden (angegeben ist jeweils die Anzahl und die Art der geschlossenen bzw. noch vorhandenen Einrichtungen)

fel). Dieses Ergebnis kann als eindeutiges Bekenntnis zum ländlichen Raum als Wohnstandort gewertet werden.

Bei den Motiven für den Verbleib werden vor allem weiche Standortfaktoren bzw. emotionale Gründe angegeben (vgl. Abb. 8). Die ruhige Lage, die schöne Landschaft oder die Tatsache, dass jemand einen großen Teil seines Lebens in der Gemeinde verbracht hat, werden in Vorpommern jeweils von mehr als $60 \%$ der Befragten genannt. In der Westeifel sind die Argumente nahezu identisch, wobei die
Eifel-Bewohner von ihrer Region noch etwas mehr überzeugt zu sein scheinen, wie die etwas höheren Werte zeigen. In der Westeifel werden zudem die Aspekte „Ich besitze hier mein eigenes Haus“, „Die Lebensqualität ist gut" und „Ich fühle mich insgesamt wohl“ von jeweils 70 bzw. $60 \%$ angeführt.

Bei den Motiven wegzuziehen gaben zunächst $30 \%$ aus beiden Regionen spontan an, es gebe keinen einzigen Grund, 
Abb. 7 Migrationsbereitschaft der befragten Einwohner. $(1=$ ich werde auf jeden Fall umziehen, $8=$ ich werde sicher nicht umziehen; Angaben je Skalaeinheit in Prozent)
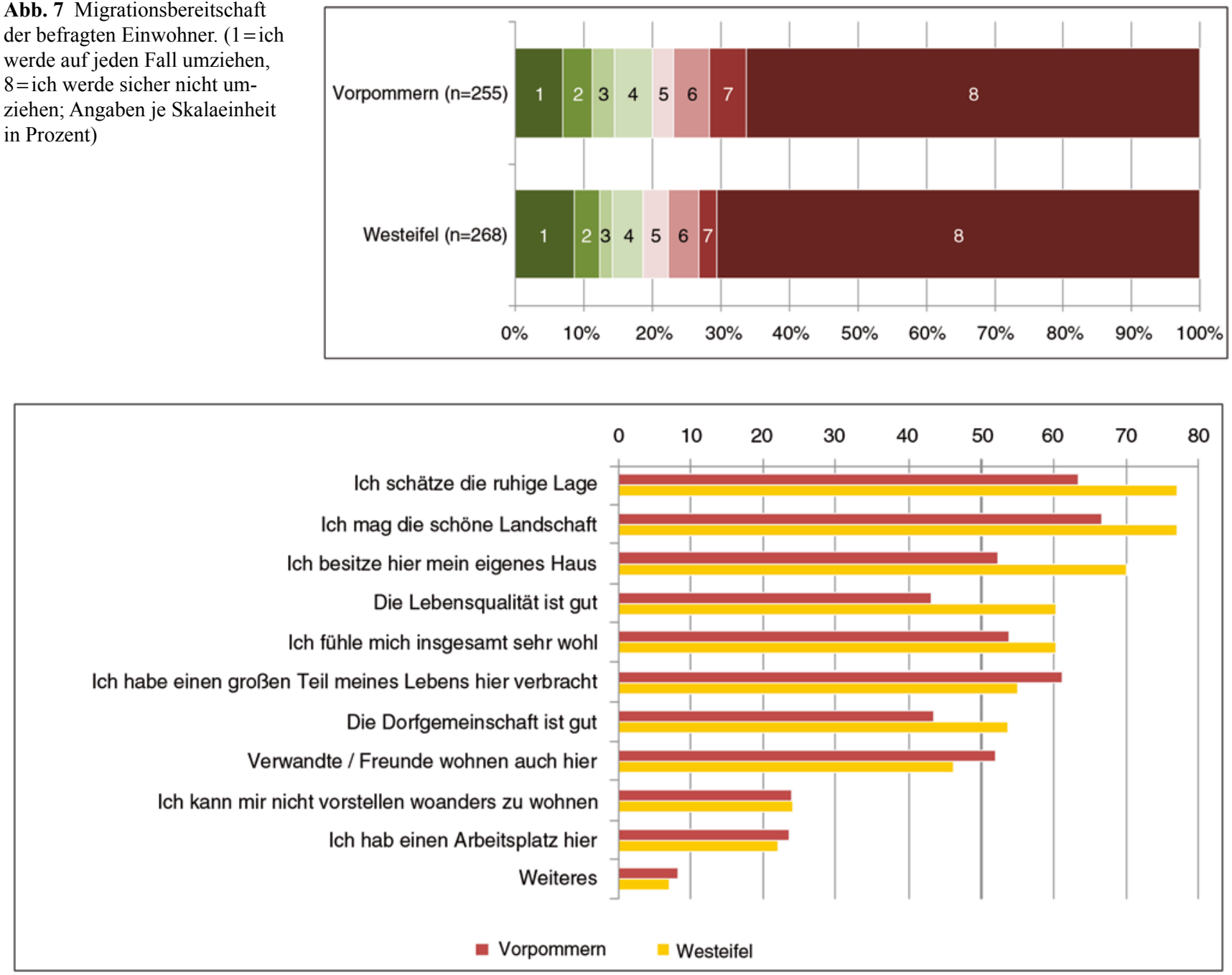

Abb. 8 Gründe der befragten Bewohner in der Region zu verbleiben. (geschlossene Frage, Mehrfachantworten, angegeben ist der Prozentsatz der Personen, die den jeweiligen Aspekt genannt haben; Westeifel: $n=270 / 1494$ Antworten; Vorpommern: $n=271 / 1334$ Antworten)

der sie zum Wegzug veranlassen könnte. ${ }^{2}$ Dieses Ergebnis kann aus zweierlei Perspektiven interpretiert werden. Zum einen könnte dies auf ein ausgesprochen großes Beharrungsvermögen der Bewohner und ihre Verbundenheit mit der Region zurückzuführen sein, was durch die Motive zum Verbleib (vgl. Abb. 8) unterstützt wird. Zum anderen kann gemutmaßt werden, dass die mobile Bevölkerung bereits abgewandert ist und die noch dort lebenden Menschen nicht mehr migrationswillig sind. ${ }^{3}$

Die Motive zum Wegzug aus der Region (vgl. Abb. 9) deuten erste entscheidende Unterschiede zwischen den Untersuchungsregionen an. In Vorpommern stechen deut-

\footnotetext{
${ }^{2}$ Diese Personen zählen zu jener Gruppe, die ganz sicher nicht umziehen möchte (vgl. Abb. 7).

${ }^{3}$ Diese Vermutung wird vor allem für die Region Vorpommern durch die Ausführungen von Weiß zur Residualbevölkerung gestützt, der er eine signifikante Sesshaftigkeit zuschreibt (vgl. Weiß 2006: 494).
}

lich arbeitsplatzgebundene Gründe wie „Keine Aussicht auf Arbeit“ (40\%) oder „Eine zu große Entfernung zum Arbeitsplatz“ (22\%) für einen potenziellen Wegzug hervor. Alle weiteren Argumente treten stark in den Hintergrund. In der Westeifel hingegen sind die Gründe sehr viel breiter verteilt. Am häufigsten wurde die Antwort genannt, dass eine ungenügende Versorgungsstruktur zum Wegzug veranlassen könnte (22\%). An zweiter Stelle wird auch hier „Keine Aussicht auf Arbeit" angeführt, allerdings nur von $17 \%$ der Befragten.

Als Zwischenfazit kann für das Segment der Gemeinsamkeiten festgehalten werden, dass es sich sowohl bei der Westeifel als auch bei Vorpommern um Räume handelt, die sich bereits seit mehreren Jahrzehnten entleeren und dass dieser Trend mit allen negativen Konsequenzen für die Bevölkerungsstruktur und den Abbau der Infrastruktur auch in Zukunft anhalten wird. Die Menschen, die auf dem Land leben, sind jedoch von ihrem Wohnstandort überzeugt und 


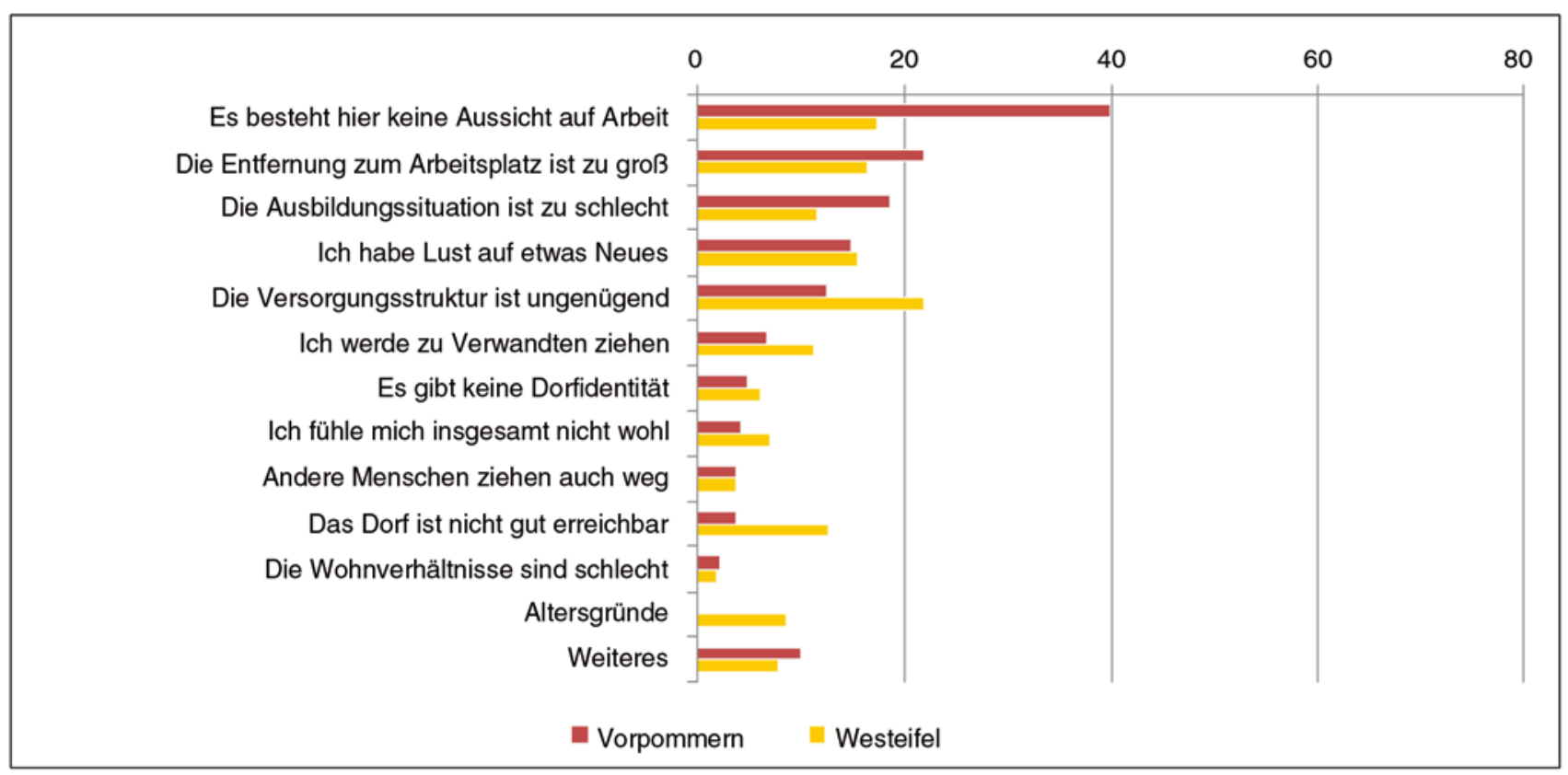

Abb. 9 Gründe der befragten Bewohner, aus der Region wegzuziehen. (geschlossene Frage, Mehrfachantworten, angegeben ist der Prozentsatz der Personen, die den jeweiligen Aspekt genannt haben; Westeifel: $n=270 / 463$ Antworten; Vorpommern: $n=271 / 468$ Antworten)

aus ihrer Sicht überwiegen die Vorteile. Das Bild der Untersuchungsregionen wird jedoch sehr viel differenzierter, wenn man die Unterschiede mit betrachtet.

\section{Unterschiede}

Zur Analyse der Unterschiede wird zunächst erneut ein Vergleich wichtiger Sekundärdaten vorgenommen, um naturräumliche, raumstrukturelle und sozioökonomische Gegensätze aufzudecken. Eine weitere Differenzierung erfolgt anhand der Aspekte Dorfgemeinschaft, Immobiliensituation und Zukunftserwartung der befragten Einwohner.

\subsection{Strukturvergleich}

Unterschiede sind zunächst hinsichtlich des Landschaftscharakters festzustellen. Während es sich beim Vorpommerschen Binnenland um eine strukturarme, ackergeprägte und offene Kulturlandschaft handelt, finden wir in der Westeifel eine stärker grünlandgeprägte und waldreiche Mittelgebirgskulturlandschaft vor. Teile der grenznahen Westeifel zählen zum Deutsch-Luxemburgischen Naturpark, im Norden hat die Westeifel zudem Anteile am Deutsch-Belgischen Naturpark Hohes Venn-Eifel. Somit ist die landschaftliche Attraktivität der Westeifel für den Tourismus größer als im eher monoton agrarisch geprägten vorpommerschen Binnenland.

Bezüglich der Zentrenerreichbarkeit und Bevölkerungsdichte stellen beide Regionen Peripherräume mit sehr geringer Dichte dar. Betrachtet man jedoch die Karte des Bundesamtes für Bauwesen und Raumordnung (2005: 20) zur Raumstruktur in Deutschland, so wird ein wesentlicher Unterschied zwischen beiden Räumen deutlich: Während Vorpommern weiträumig von weiteren Peripherräumen mit sehr geringer Dichte umgeben ist, ist die Westeifel in verschiedene Zwischen- (Großraum Trier, Saartal) und Zentralräume (Rhein-Main, Koblenz, Aachen, Rhein-Ruhr) eingebettet.

Ein weiterer wichtiger Unterschied ist in der Landwirtschaft zu sehen. Während in der Westeifel Familienbetriebe mit durchschnittlich 41 ha überwiegen und mit ihrer Infrastruktur die Dörfer prägen, wird die Landwirtschaft in Vorpommern vorwiegend von überregional oder sogar international organisierten Großbetrieben mit durchschnittlich 284 ha dominiert und völlig losgelöst von den Dörfern organisiert (vgl. Hauss/Land/Willisch 2006: 34; Beetz/ Huning/Plieninger 2008: 301; Statistisches Amt Mecklenburg-Vorpommern 2007; Statistisches Landesamt Rheinland-Pfalz 2009).

Darüber hinaus sind bedeutsame sozioökonomische Gegensätze zu konstatieren. Der regionalwirtschaftliche Entwicklungsstand wird für die Westeifel als „,durchschnittlich“ und für Vorpommern als ,deutlich unterdurchschnittlich“ eingestuft (vgl. Bundesamt für Bauwesen und Raumordnung 2005: 151; Glaser/Gebhardt/Schenk 2007: 74). Dies schlägt sich auch drastisch in den Arbeitslosenzahlen nieder: Die Quote schwankt in den vorpommerschen Landkreisen zwischen 17 und $23 \%$ und im Eifelkreis Bitburg-Prüm liegt sie bei nur knapp $5 \%$. Dies ist in erster Linie auf die Nähe zu 
Luxemburg zurückzuführen, wo zahlreiche Bewohner der Westeifel einen gut bezahlten Arbeitsplatz gefunden haben. Auch beim Schuldenstand befindet sich die Westeifel in einer besseren Position. Die Untersuchungsgemeinden sind hier mit durchschnittlich 302 Euro pro Einwohner verschuldet, in Vorpommern dagegen mit 1.770 Euro (vgl. Statistisches Amt Mecklenburg-Vorpommern 2007; Statistisches Landesamt Rheinland-Pfalz 2009).

Aus dieser Gegenüberstellung wird bereits deutlich, dass die Rahmenbedingungen, innerhalb derer der demographische Wandel wirksam wird, sehr verschieden sind, und dass sich die Westeifel in einer weitaus stabileren Position befindet.

\subsection{Die Dorfgemeinschaften}

Betrachtet man die Dorfgemeinschaften, so zeigen sich weitere eklatante Gegensätze zwischen den Untersuchungsräumen. Die Einwohner wurden im Rahmen der Befragung gebeten, einigen Aussagen zur Dorfgemeinschaft zuzustimmen oder diese abzulehnen (vgl. Abb. 10).

Mehr als die Hälfte der Befragten in Vorpommern stimmt der Aussage zu, dass sich das Zusammenleben in den letzten zehn Jahren verschlechtert hat. Nur ein Fünftel der Befragten in der Westeifel ist dieser Ansicht. Darüber hinaus bestätigt fast die Hälfte der Befragten in Vorpommern, dass es soziale Spannungen im Dorf gibt, und knapp ein Viertel sagt aus, dass sich die Bewohner aus dem Weg gehen. In der Westeifel bewegen sich die Aussagewerte in beiden Segmenten deutlich darunter. Schließlich bejahen nur $64 \%$ in Vorpommern, dass der Zusammenhalt der Dorfgemeinschaft funktioniert. In der Westeifel sind dagegen $83 \%$ dieser Ansicht.

Bezieht man die Aussagen der Bürgermeister mit ein, wird das Bild der Dorfgemeinschaften in Vorpommern noch weiter verschärft. Die folgenden Zitate verdeutlichen die Situation:

- „Egoismus und Neid haben zugenommen“ (Koblentz)

- "Viele Menschen [...] verfallen dem Alkoholismus“ (Schönfeld)

- Es gibt „negative Emotionen, Unsicherheit und wenig Freude" (Glasow)

- „Jeder kämpft für sich“ (Gültz)

Nach Aussage der Experten aus Vorpommern leiden in den Dörfern vor allem traditionelle Werte und kommunales Engagement unter dieser Situation. Grundtenor der Gespräche war, dass das Dorfleben durch ein reduziertes Gemeinschaftsleben und eine Auflösung des solidarischen Verhaltens geprägt ist (vgl. hierzu auch Herrenknecht 1995: 54 ff.). Aus der Perspektive der möglichen Umkehrung des Schrumpfungsprozesses ist diese Tatsache vor allem deshalb problematisch, da von solchen Dorfgemeinschaften nur selten positive Impulse und Eigeninitiative ausgehen. Gerade an diesem Punkt bietet sich regressiven Degradationspro-

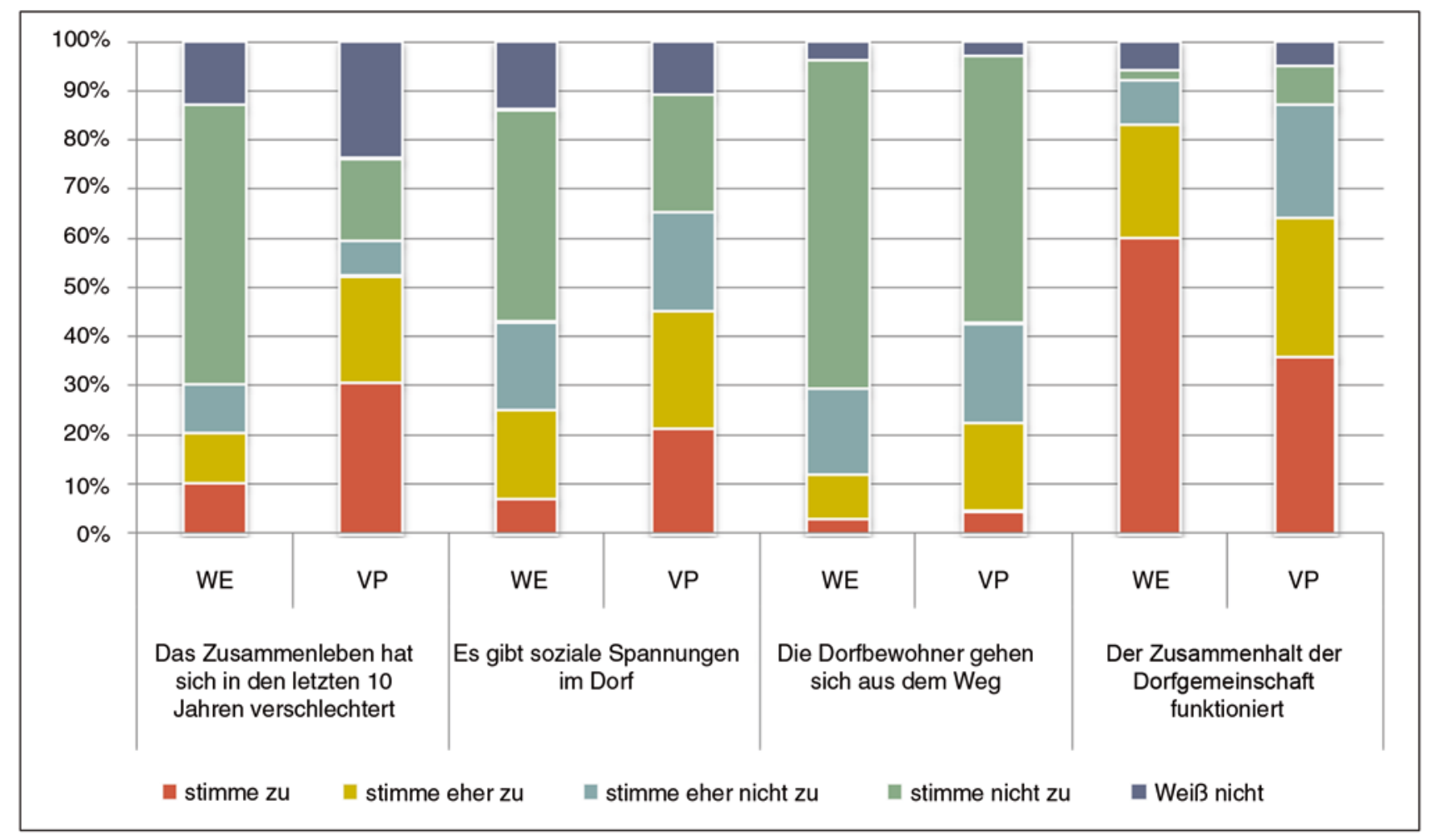

Abb. 10 Beurteilung der Dorfgemeinschaft durch die Bewohner. (Westeifel (WE): $n=270$; Vorpommern (VP): $n=271$ ) 
Tab. 1 Bewertung der Grundstimmung in den Untersuchungsgemeinden durch die Bürgermeister

\begin{tabular}{lcl}
\hline & $\begin{array}{l}\text { Vorpommern } \\
(11 \text { Gemeinden })\end{array}$ & $\begin{array}{l}\text { Westeifel } \\
(22 \text { Gemeinden })\end{array}$ \\
\hline positiv & $9,1 \%$ & $54,5 \%$ \\
mittelmäßig & $18,2 \%$ & $31,8 \%$ \\
negativ & $72,7 \%$ & $13,6 \%$ \\
\hline
\end{tabular}

zessen eine große Angriffsfläche. Zahlreiche Studien haben belegt, dass gerade die Eigeninitiative der Menschen vor Ort unerlässlich ist, um ein Dorf wieder zu beleben (vgl. stellvertretend Stöber 2006).

In der Westeifel hingegen stellen die Dorfgemeinschaften kein Thema dar, zu denen die Bürgermeister in besonderer Weise Stellung bezogen hätten. Es existieren lediglich in drei der Untersuchungsgemeinden Streitigkeiten. In einem Fall haben diese einen im Vergleich zu Vorpommern trivialen Grund, nämlich den Bau einer Grillhütte. In zwei weiteren Fällen geben Flurbereinigungsverfahren Anlass zu Kontroversen.

Dies spiegelt sich auch in der qualitativen Bewertung der Grundstimmung der Untersuchungsgemeinden wider, die durch die Bürgermeister vorgenommen wurde. In der Westeifel wird sie für $54,5 \%$ der Gemeinden als positiv und für weitere $31,8 \%$ als mittelmäßig eingestuft. In Vorpommern hingegen wird sie für $72,7 \%$ der Gemeinden als negativ bewertet (vgl. Tab. 1).

\subsection{Immobiliensituation}

In beiden Regionen hat sich der Immobilienmarkt in den beiden letzten Jahrzehnten stark gewandelt. Jedoch laufen die Entwicklungen in ihrer Grundtendenz in entgegengesetzte Richtungen.

In Vorpommern ist ein deutlicher Angebotsüberhang auf dem Wohnungsmarkt zu konstatieren, was sich in verschiedenen Expertenmeinungen folgendermaßen niederschlägt:

- „Die Auswirkungen des demographischen Wandels sind stark spürbar: der Bevölkerungsrückgang zieht Preisabschläge bis zu $50 \%$ nach sich“ (Immobilienmakler Neubrandenburg).

- „Durch die Abwanderung der jungen Menschen kommt es speziell in den ländlichen Regionen zum Leerstand. Nur durch Abrisse von Häusern können die Mietpreise erhalten werden“ (Immobilienmakler Neubrandenburg).

- „Die Leerstände sind oftmals in einem solch desolaten Zustand, dass nur noch ein Abriss in Betracht gezogen werden kann" (Bürgermeisterin Voigtsdorf).

Betrachtet man das Erscheinungsbild der Untersuchungsgemeinden in Vorpommern, so ist offensichtlich, dass Leerstände das Erscheinungsbild der Dörfer beeinträchtigen und eine Atmosphäre von Verfall und Perspektivlosigkeit suggerieren (vgl. Abb. 11 und 12). Es handelt sich zwar noch

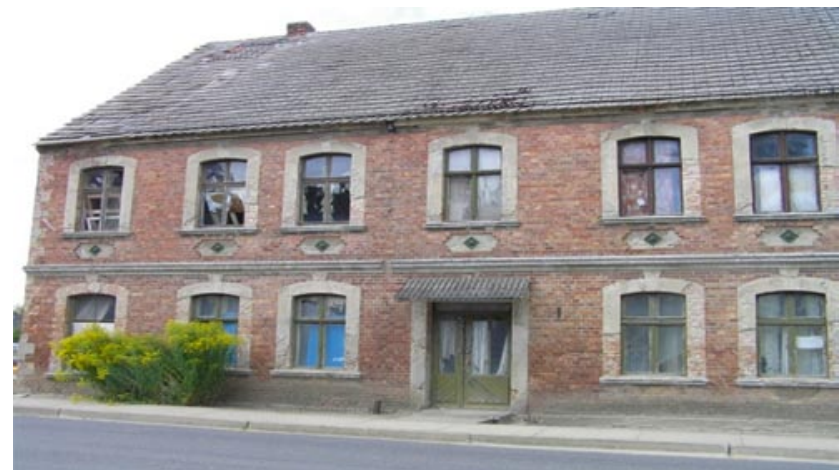

Abb. 11 Verlassenes Haus mit eingeschlagenen Fenstern in Grammentin (Vorpommern)

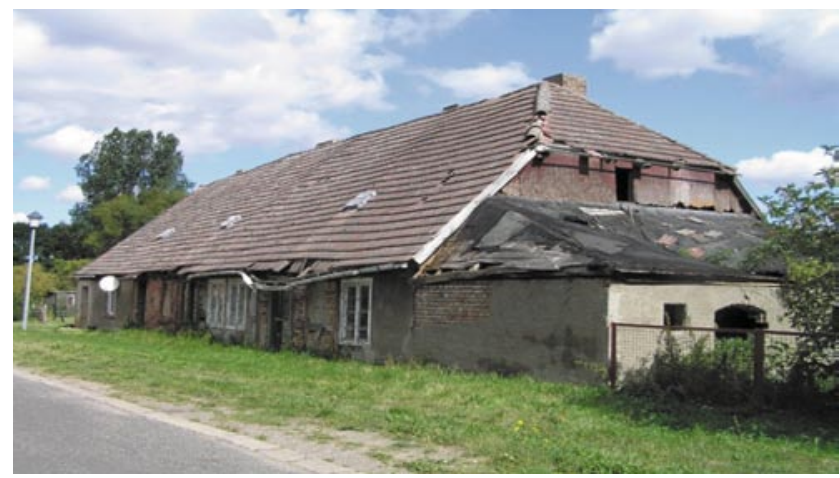

Abb. 12 Verfallendes Haus in Kruckow/Unnode (Vorpommern)

um Einzelobjekte, aber der Niedergang ist auffällig und in nahezu allen Dörfern präsent.

Auch in der Westeifel sind durchaus verfallende Häuser in den Dörfern vorzufinden, wobei diese jedoch deutlich weniger verbreitet sind. Ein Prozess, der für die Eifel weitaus charakteristischer ist, ist die Renovierung von Häusern, wodurch Immobilien im Dorfkern erhalten werden (vgl. Abb. 13 und 14). Darüber hinaus sind auch Neubauten von Einfamilienhäusern zu beobachten.

Die Erklärung hierfür bieten die folgenden Äußerungen:

- „Wenn ein Haus leer steht, wird es bedingt durch die Grenznähe sofort neu bezogen“ (Bürgermeister Sevenig/Our).

- „Leer stehende Häuser sind schnell verkauft“ (Bürgermeister Zweifelscheid).

- „Je schneller in Luxemburg, desto teurer das Grundstück“ (Hentschel 2008).

Es sind insbesondere Personen aus den Nachbarländern Luxemburg und Niederlande, aber auch aus dem Großraum Köln-Bonn, die alte Häuser in der Westeifel aufkaufen und renovieren oder neu bauen. Dabei sind nach Aussage der Bürgermeister vor allem zwei Gruppen voneinander $\mathrm{zu}$ unterscheiden. Die erste umfasst Altersruhesitzmigranten, die die Landschaft und den Mittelgebirgscharakter 


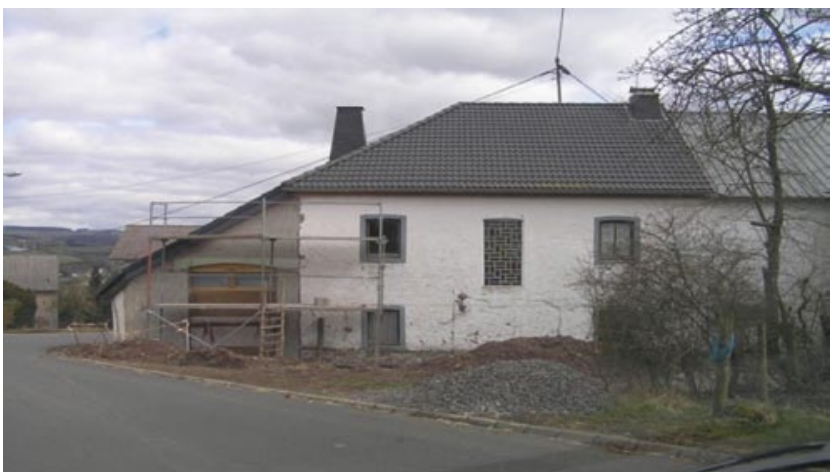

Abb. 13 In Renovierung befindliches Haus in Kinzenburg (Westeifel)

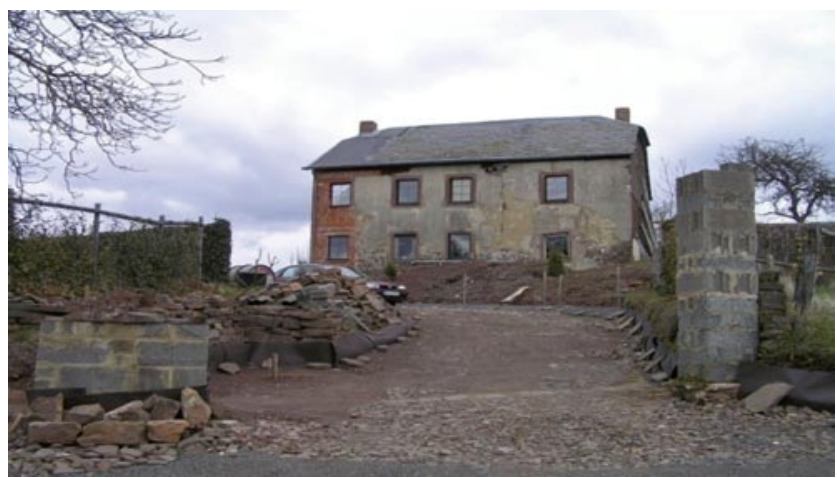

Abb. 14 In Renovierung befindliches Haus in Lascheid (Westeifel)

schätzen. Die zweite Gruppe wird von Zuwanderern aus Luxemburg gebildet, die mit ihren Familien aufgrund der niedrigen Grundstückspreise in die Westeifel ziehen. Dass dieser Prozess bewusst forciert wird, ist an den Werbeplakaten für neue Wohnbaugebiete zu erkennen. Diese richten sich explizit, zum Teil auch in französischer Sprache, an die wohlhabende Luxemburger Klientel. Allerdings können nach Aussage der befragten Experten von diesem „Luxemburg-Effekt" lediglich Dörfer in Grenznähe profitieren. $\mathrm{Ab}$ einer Entfernung von 15 bis 20 Kilometern zur Grenze ebbt das Phänomen deutlich ab.

Während also in Vorpommern die Immobiliensituation einen deutlichen Indikator für die aktuell ablaufenden Regressions- und Entleerungsprozesse darstellt, können in der Westeifel frei werdende Immobilien vielerorts an Zuwanderer und Altersruhesitzmigranten verkauft werden, wodurch die ländlichen Siedlungen belebt und stabilisiert werden.

\subsection{Zukunftserwartungen}

Als letzten Aspekt des Vergleichs werden die Zukunftserwartungen der Befragten betrachtet. Die Bewohner wurden gebeten, einzuschätzen, wie sich die Gesamtsituation ihrer Gemeinde in Zukunft entwickeln wird (vgl. Abb. 15). Insgesamt $56 \%$ der Befragten aus Vorpommern befürchten eine Verschlechterung, wobei $13 \%$ sogar davon ausgehen, dass sich die Situation erheblich verschlechtern wird. Hingegen erwarten nur $43 \%$ der Befragten in der Westeifel eine Verschlechterung. Knapp die Hälfte geht hier davon aus, dass alles bleibt wie es ist. Die Aussage, dass sich die Situation verbessern wird, hat in keiner der beiden Regionen große Zustimmung gefunden. Hier zeigt sich in der Westeifel also eine deutlich positivere Einstellung.

Bei der Benennung der größten Probleme (vgl. Tab. 2), die es zu bewältigen gilt, gehen die Meinungen ebenfalls auseinander. In Vorpommern benannten 70 \% der Befragten „Fehlende Arbeitsplätze und Arbeitslosigkeit“ als größtes Problem der Region. Dieser für Vorpommern derart herausstechende Aspekt findet in der Westeifel hingegen kaum

Abb. 15 Zukunftserwartungen der befragten Bewohner: Aussagen zur zukünftigen Entwicklung der Gesamtsituation in der Gemeinde

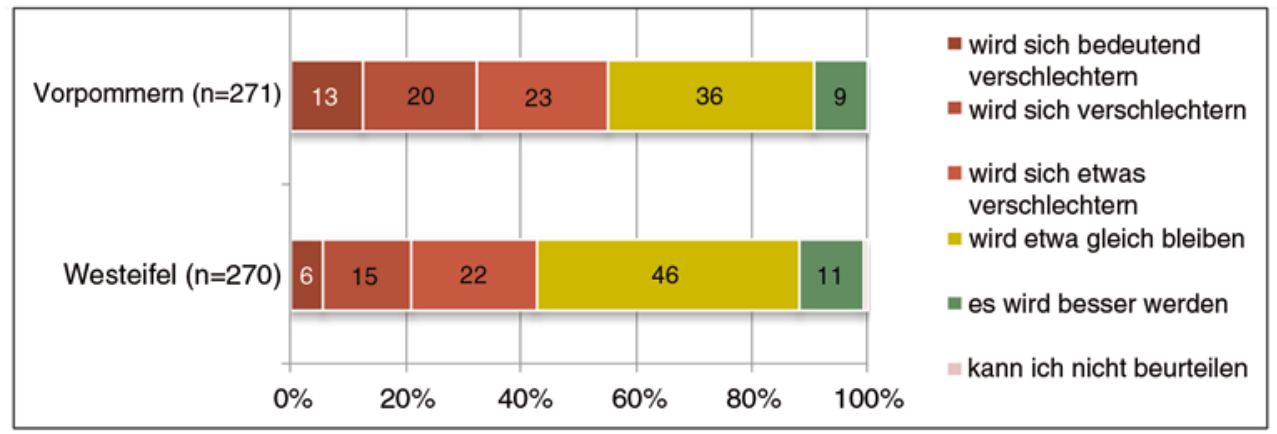

Tab. 2 Die größten Probleme in der Region aus Sicht der Befragten (Angaben in Prozent). (offene Frage, Mehrfachantworten, angegeben ist der Prozentsatz der Personen, die den jeweiligen Aspekt genannt haben; Westeifel: $n=270 / 311$ Antworten; Vorpommern: $n=271 / 415$ Antworten)

\begin{tabular}{llll}
\hline Vorpommern & & Westeifel & \\
\hline Fehlende Arbeitsplätze/Arbeitslosigkeit & 70 & Demographischer Wandel & 25 \\
Infrastruktursituation & 21 & Dorfgemeinschaft & 19 \\
Demographischer Wandel & 14 & Infrastruktursituation & 13 \\
\hline
\end{tabular}


Beachtung. Weit abgeschlagen folgen in Vorpommern die Infrastruktursituation sowie die Abwanderung und Alterung der Gesellschaft. Die Westeifel-Befragten sind weniger eindeutig bei der Problembenennung. Bei ihnen führt der demographische Wandel die Liste an, der von einem Viertel der Befragten als Problem genannt wird. Darauf folgen die Dorfgemeinschaft und die Infrastruktursituation. Die Sorge um die Dorfgemeinschaft bezieht sich auf die Überalterung und das Fehlen der Kinder, was von vielen Einwohnern als Befürchtung geäußert wird.

Weiterhin sollten die Befragten einschätzen, wie sich die Bevölkerungszahl ihrer Gemeinde in den nächsten 25 Jahren verändern wird. Auch hier ist in der Westeifel ein größerer Optimismus festzustellen; denn $85 \%$ der Befragten in Vorpommern aber nur $68 \%$ der Westeifel-Befragten erwarten eine weitere Abnahme. In der Eifel gehen sogar $17 \%$ von einer Zunahme der Bevölkerungszahl aus; in Vorpommern sind nur $7 \%$ dieser Ansicht.

Generell lässt sich festhalten, dass die Befragten in Vorpommern ihre Zukunft pessimistischer einschätzen als die Eifelbewohner. Ihre Selbstwahrnehmung ist negativ geprägt, und die Chancen, ideale Lebensentwürfe zu verwirklichen, werden als recht gering eingeschätzt.

\section{Ergebnis der vergleichenden Analyse}

Betrachtet man die Gemeinsamkeiten und Gegensätze der beiden Untersuchungsregionen, so kann folgendes Ergebnis festgehalten werden. Gemeinsamkeiten bestehen hinsichtlich der dezentralen, kleinteiligen Siedlungsstruktur und dem weitständigen Netz Zentraler Orte. Sowohl die Westeifel als auch Vorpommern sind von zwei wichtigen Komponenten des demographischen Wandels erfasst, nämlich einer altersstrukturellen Verschiebung mit unverkennbarer Überalterungstendenz und einem deutlichen Bevölkerungsrückgang. Diese Faktoren haben sich bereits in einem Abbau der Infrastruktur niedergeschlagen. Aber trotz der daraus resultierenden Beeinträchtigung der Lebensqualität ist der größte Teil der Bevölkerung keineswegs geneigt abzuwandern. Diese Gemeinsamkeiten bilden zunächst einen Rahmen, innerhalb dessen zwischen den beiden Regionen weiter differenziert werden muss. Zu diesem Zweck sollen die eingangs formulierten Hypothesen erneut aufgegriffen werden.

Die erste Annahme, dass die Brisanz des demographischen Wandels durch die großräumliche Einbettung einer Region verschärft oder abgeschwächt wird, kann anhand des Vergleiches bestätigt werden. Eine wichtige Determinante ist nämlich das Ausmaß der Peripheralität und die Lage der Regionen zu anderen wirtschaftlichen Kernräumen Europas. Diese Lage bedingt für die Westeifel bessere und für Vorpommern schlechtere Voraussetzungen für die Teilhabe an positiven Entwicklungseffekten. Beide Regionen liegen zwar auf einer Achse von Problemregionen, die sich von Vorpommern über die Eifel, das französische Zentralmassiv und Nordwestspanien zieht, und sie zählen damit zu der Gruppe benachteiligter Räume in Europa (vgl. Diercke Weltatlas 2008: 85). Aber die Westeifel liegt zusätzlich in unmittelbarer Nähe zu verdichteten und wirtschaftsstarken Regionen und kann somit durch Migrations- und Pendelbewegungen von ihren Nachbarländern profitieren. Die Westeifel selbst ist zwar als „Schwächezone“ zu bezeichnen, doch erscheint sie großräumig deutlich besser eingebettet als Vorpommern.

Die Lage im Grenzraum hat den entscheidenden Effekt, dass die in der Westeifel lebenden Menschen auch in außerländlichen Arbeitsbereichen, etwa dem Luxemburger Bankensektor, eine Anstellung finden und daher nicht durch berufliche Gründe zur Abwanderung gezwungen sind. Begünstigt wird dies durch die praktisch fehlende Sprachbarriere zwischen Deutschland und Luxemburg. Die unmittelbare Nähe der Nachbarländer Luxemburg, Belgien (mit der Deutschsprachigen Gemeinschaft) und Niederlande mit den Euregios Maas-Rhein und SaarLorLuxRhein sowie die Nachbarschaft zu den Metropolregionen Rhein-Ruhr, Frankfurt/Rhein-Main und dem Verdichtungsraum Saar ermöglicht zudem in Kombination mit der attraktiven, abwechslungsreichen Mittelgebirgslandschaft Zuwanderung in die Westeifel. Diese wird durch die günstigen Immobilienpreise und die Naturnähe initiiert. Der entscheidende Standortfaktor der Westeifel ist somit die Nähe zu bevölkerungsreichen und zum Teil auch prosperierenden Regionen. Als problematisch ist jedoch die dadurch bedingte Abhängigkeit der Westeifel von exogenen positiven Einflüssen zu erachten. Sollte Luxemburg als Arbeitsmarkt wegbrechen und das Interesse der Nachbarländer an der Westeifel als Wohn- und Freizeitraum nachlassen, so kann dies nachteilige und entwicklungshemmende Auswirkungen auf die Region haben.

Vorpommern hingegen ist bislang noch nicht in der Situation, von positiven Einflüssen seiner Nachbarräume profitieren zu können. Die Region liegt zwar in unmittelbarer Nachbarschaft zum Großraum Stettin mit mittlerweile rund 500.000 Einwohnern; aber dies hat sich bislang noch nicht spürbar ausgewirkt. Hoffnungen bestehen indessen durch die verkehrsgeographisch günstige Lage von Stettin, dessen wirtschaftliche Basis durch die maritime Industrie, seinen Wissenschaftssektor mit den Hochschulen und nicht zuletzt seinen touristisch attraktiven Charakter. Wenn es gelingt, Stettin zu einem dynamischen Zentrum zu entwickeln, sind vermutlich auch deutlich positive Veränderungen für das deutsche Umland zu erwarten (vgl. Maack/Grundmann/ Kreft et al. 2005).

Auch der zweite Teil der Hypothese, der besagt, dass die Brisanz des demographischen Wandels durch die Ausgangssituation einer Region mit ihren spezifischen naturräumlichen, 
ökonomischen, gesellschaftlichen und siedlungsgeographischen Besonderheiten verschärft oder abgeschwächt wird, kann anhand des Vergleiches bestätigt werden. Strukturschwache Räume wie die Westeifel, die von Vorteilen wie naturräumlichen Potenzialen oder geringer Arbeitslosigkeit profitieren können, besitzen weitreichendere Möglichkeiten, auf den demographischen Wandel zu reagieren. Zudem werden die Auswirkungen des Wandels durch positive Einflüsse zum Teil kompensiert. Im vorpommerschen Binnenland hingegen, wo zahlreiche Problemlagen in einer Abwärtsspirale kumulieren, kann dem demographischen Wandel wenig entgegengesetzt werden. Mit einer medizinischen Metapher gesprochen könnte man auch sagen, dass die Abwehrkräfte der Region in Anbetracht der Vielzahl der Probleme erschöpft sind. In Vorpommern bestehen wirtschaftliche Rahmenbedingungen, die ein nachhaltiges Wirksamwerden des demographischen Wandels fördern, wodurch fortwährend die Innovations- und Leistungsfähigkeit der Region geschwächt und Peripherisierungsprozesse genährt werden (vgl. Keim 2006). Die wirtschaftlichen und gesellschaftlichen Probleme in Vorpommern werden durch den demographischen Wandel jedoch lediglich verstärkt und nicht ursächlich durch ihn hervorgebracht. Die eigentliche Ursache der wirtschaftlichen und gesellschaftlichen Problemlagen ist in der Entwicklung seit der Wiedervereinigung zu suchen. Ein wesentlicher Teil der demographischen Entwicklung ist somit selbst teils Folge und teils Moment der Umbrüche seit 1989 (vgl. Land/Willisch 2006: 54-55; Hübler/Kujath 2007: 10 ff.). Daher besteht aber auch die Gefahr, dass durch eine Überhöhung des Phänomens demographischer Wandel die eigentlichen Probleme dieses Raumes aus dem Blickfeld geraten (vgl. Beetz/Huning/Plieninger 2008: 306).

Insgesamt betrachtet, befindet sich die Westeifel in einer stabileren Situation. Zwar wird auch diese Region weiterhin altern, schrumpfen und mit einem geringen $\mathrm{Ma} ß$ an Versorgungsinfrastruktur zurechtkommen müssen, aber der demographische Wandel wird sich weitaus weniger destruktiv auswirken als in Vorpommern.

\section{Entwicklungsperspektiven}

Die Analyse der Strukturen und Probleme in den Untersuchungsregionen Vorpommern und Westeifel macht deutlich, dass der demographische Wandel sehr unterschiedliche und auch verschieden starke Handlungsbedarfe erfordert. Für jede Region sind spezifische Lösungswege zu beschreiten, die auf die besonderen Strukturen, Probleme und deren Genese abgestimmt sind. Das ,Abgehängtsein' von zentralen Entwicklungen schafft dabei aber auch Bedingungen für räumlich begrenzte Regelsysteme mit Chancenfür experimentelle Nutzungen und Lebensformen ohne den Druck der hegemonialen Zentralentwicklungen (vgl. Keim 2006: 5-6). In diesem Kontext ist nach regionalen Ansatzpunkten für Gegentrends bzw. Selbstbehauptungen zu suchen und es sind vor allem auf regionalpolitischer Ebene Lösungskonzepte zu entwickeln (vgl. Keim 2006: 5-6). Die Definition konkreter Handlungsfelder setzt jedoch als Grundlage „ein langfristiges, strategisches Leitbild, ein plausibles Bild einer erstrebenswerten, machbaren Zukunft für die Region“" (Kocks 2004: 31) voraus. Die darin formulierten Entwicklungsperspektiven sind durch entsprechende Anpassungsmaßnahmen zu erreichen.

\subsection{Westeifel}

Das endogene Potenzial der Westeifel legt eine Ausrichtung auf die Segmente Ruhesitzmigration, Naherholung und nachhaltige Landschaftsentwicklung nahe. Die Erholungs- und Freizeitfunktion vermag ein eigenes, stabiles und entwicklungsträchtiges Profil herbeizuführen, wobei die Zielgruppe älterer Menschen einen Schwerpunkt bilden sollte. Das Beispiel Westeifel zeigt, dass Siedlungsregression unter bestimmten Bedingungen Freiräume für neue Nutzungen und neue Bevölkerungsgruppen schaffen kann: Die aufgrund der Peripherisierung niedrigen Grundstücksund Immobilienpreise stellen zusammen mit der Lagegunst der Westeifel Vorteile für Familien aus Luxemburg dar, da sie günstig und in der Nähe zu ihrer Heimat ein Eigenheim erwerben oder bauen können. Zum anderen wird die Region durch die naturnahe Kulturlandschaft, die dünne Besiedlungsdichte und die landschaftlichen Reize der Mittelgebirgsregion als Zielgebiet für Erholungssuchende und Altersruhesitzmigranten begünstigt. Für die Westeifel stellt daher insbesondere die Nähe zu Agglomerationsräumen einen entscheidenden Standortfaktor dar, durch den stabilisierende Verflechtungen mit Stadt- und Metropolregionen initiiert werden konnten. Von dieser Nähe profitieren zum einen die freizeit- und tourismusorientierten Besucher, zum anderen aber auch die arbeitsweltlich motivierten Auspendler. Gerade durch die Auspendlerströme wird der positive Effekt erzielt, dass Kaufkraft in der Region verbleibt, da die an anderer Stelle erzielten Einkommen zumindest zum Teil in der Heimatregion ausgegeben werden.

Insbesondere durch die Ausgleichs- und Schutzfunktion des ländlichen Raumes der Westeifel sind auch in Zukunft Chancen zum Peripherisierungsabbau zu erwarten. Die die Westeifel in einem Radius von bis zu 100 Kilometer umgebenden Räume haben zudem weitere Potenziale durch ihre Erholungs- und ökologischen Werte. So findet beispielsweise die attraktive Mittelgebirgslandschaft der Westeifel ihre Fortsetzung im deutsch-belgischen Naturpark Hohes Venn-Eifel, im Nationalpark Eifel und im Naturpark SaarHunsrück. Diese Nähe birgt große Potenziale für eine nachhaltige Entwicklung und eine versöhnende Koexistenz von 
Mensch und Natur mit einem stabilisierten Siedlungssystem und ökologisch wertvollen Landschaften. Auf dieser Basis kann nicht zuletzt gezielt ein positives ,grünes' Image entwickelt werden. Wichtige Voraussetzungen dafür sind eine entsprechende Dienstleistungs- und Handelsinfrastruktur, die sich auch im Bereich der medizinischen Fürsorge speziell auf ältere Menschen und Ruhesitzmigranten einstellt, ein attraktives Gastgewerbe und Freizeiteinrichtungen sowie eine gute (touristisch interessante) Erreichbarkeit der Region (vgl. hierzu auch Keim 2006: 5). Dabei ist auch insbesondere der Erhalt der Versorgungsinfrastruktur für die Attraktivität der Westeifel als Standort für Freizeitwohnund Altersruhesitze bedeutsam.

\subsection{Vorpommern}

Vorpommern hingegen unterliegt auf mehreren Ebenen einem Peripherisierungsprozess und ist durch eine hohe Abhängigkeit gekennzeichnet. Es besteht die Gefahr, dass die Region um einer vermeintlich wirtschaftlichen Effizienz willen voreilig abgeschrieben wird. Viele Beispiele haben jedoch gezeigt, dass ,,als hoffnungslos zurückgeblieben eingestufte Räume sich wider Erwarten hervorragend entwickeln“ (Heinrichs 2006: 653). Die staatlichen Institutionen sind daher gefordert, Möglichkeiten für eine eigenständige und selbstverantwortliche Regionalentwicklung zu eröffnen, die auf folgende Schwerpunkte ausgerichtet ist:

- Entwicklung des sozioökonomischen Potenzials zur Erhöhung der Verwirklichungschancen der dort lebenden Menschen, vor allem auch der jüngeren und erwerbstätigen Bevölkerungsgruppen,

- nachhaltige Nutzung der naturräumlichen, kulturellen und ökonomischen Ressourcen,

- gezielte Förderung ökonomischer und kultureller Impulse durch den Aufbau eines positiven regionalen Images (vgl. Akademie für Raumforschung und Landesplanung 2008: 1).

Vorpommern braucht ein eigenes, den Besonderheiten und Stärken entsprechendes Profil nachhaltiger wirtschaftlicher und sozialer Entwicklung. Hier sind neue Leitorientierungen gefragt, die nicht zuletzt eine mögliche Rolle als „Verbindungsregion" " zwischen West- und Osteuropa reflektieren (vgl. Reißig 2003:24). Eine veränderte geostrategische Lage, wie sie sich für Vorpommern durch die EU-Osterweiterung ergeben hat, und eine gewandelte wirtschaftliche Situation können neue Spielräume eröffnen, die heute womöglich noch gar nicht in Gänze erkennbar sind (vgl. Akademie für Raumforschung und Landesplanung 2008: 6). Eine Chance hierfür liegt sicherlich in einer Stärkung der Stadt-UmlandVerflechtungen von Stettin.

Ansätze für positive Entwicklungen sind indessen auch - trotz der im Vergleich zur Westeifel geringen Potenziale
- im Bereich Freizeit und Erholung gegeben. Nicht nur die gewachsene Kulturlandschaft mit ihren Schlössern, Gutshäusern, Seen- und Flusslandschaften bietet hierfür Ansatzpunkte. Auch die Einbettung von Vorpommern zwischen den Naturparken Usedom und Feldberg, dem Müritz Nationalpark und der touristisch attraktiven Ostseeküste legt derartige Überlegungen nahe. Durch die Autobahnen A11, A19 und A20 sind nämlich die Ballungsräume Hamburg, Berlin und Stettin sehr gut erreichbar. Die sich beiderseits dieser Verkehrskorridore erstreckenden Gebiete könnten sich aufgrund ihres niedrigen Preisniveaus und ihrer Zentrumsnähe als interessante Alternative zu den überlasteten und hochpreisigen Tourismusräumen entwickeln (vgl. Klüter 2009: 6). Erste Ansätze einer pionierhaften Besiedlung sich entleerender Dörfer sind bereits erkennbar, so zum Beispiel in Klein Jasedow, wo eine Initiative von 17 Personen unter anderem die „Europäische Akademie der Heilenden Künste“ erfolgreich etabliert hat.

Ein neuer zukunftsträchtiger Wirtschaftsbereich könnte sich nicht zuletzt im Energiesektor in Kombination von Wind- und Biomasseenergie entwickeln, indem sich das landwirtschaftlich geprägte Vorpommern gemeinsam mit anderen ostdeutschen Regionen als „Vorreiter einer aus ökologischen Gründen zwingend notwendigen Energiewende“ (Keppler/Land/Laufer et al. 2009: 2) etabliert. Eine solche „ökologische Reindustrialisierung“ (Keppler/Land/Laufer et al. 2009: 2) könnte realistische Chancen für eine selbsttragende wirtschaftliche Entwicklung schaffen. Dies impliziert nicht nur die Erzeugung regenerativer Energien selbst, sondern auch den Auf- und Ausbau von Kapazitäten in Forschung, Entwicklung, Industrieanlagen und entsprechenden Dienstleistungsangeboten. Auf diese Weise könnten neue Wertschöpfungsketten aufgebaut, Arbeitsplätze geschaffen und ein entscheidender Beitrag zur Vermeidung des Klimawandels geleistet werden. Bereits existierende erfolgreiche Projekte wie in Varchentin (Landkreis Müritz), das sich mit einem neuen Energiekonzept unabhängig gemacht hat, könnten als Aufhänger genutzt werden (vgl. Land/Thie 2009: $115 \mathrm{ff}$.).

Ein wichtiges Moment im Kontext der eigenständigen und selbstverantwortlichen Regionalentwicklung ist zudem der Abbau der Distanz zwischen den die Entscheidungen treffenden Institutionen und den betroffenen Bürgern, indem die in der Region lebenden Menschen in die Planung einbezogen werden (vgl. Beetz/Huning/Plieninger 2008: 296; Kocks 2004: 31). Stöber (2006: 21 ff.) plädiert daher für Entwicklungsstrukturen, an denen Bürger mitgestalten und mitarbeiten können, die eine offene Kommunikation ermöglichen und sich über innovative Ansätze neue Identitäten schaffen. Die sich aus der Analyse ergebende Tatsache, dass die Menschen gerne im ländlichen Raum leben und dessen Qualitäten schätzen, legt die Vermutung nahe, dass sich die Bewohner auch für plausible und überzeugende 
Anpassungsmaßnahmen engagieren würden. Bürgerschaftliches Engagement kann sinnstiftend und identitätsfördernd wirken, wodurch zusätzlich die Dorfgemeinschaften auf eine neue, kooperative und solidarische Basis gestellt würden. Auf diese Weise könnten den Menschen reale Chancen eröffnet und durch eine positive Selbstwahrnehmung neue Tatkraft und mutvolles Handeln in die Region gebracht werden.

Abschließend ist festzuhalten, dass es auch trotz gezielter Anpassungsmaßnahmen im Kontext des demographischen Wandels mit sinkender Bevölkerungszahl und räumlich selektiver Abwanderung immer Regressionsprozesse geben wird. Es ist daher wichtig, beständig Lösungen zu erproben und sich gleichzeitig damit zu arrangieren, dass Peripherisierung stets mit Verlusten verbunden ist. Es geht vordringlich um die Entwicklung und Umsetzung situationsbezogener, innovativer Lösungen und Konzepte, die nicht dogmatischen Standardvorgaben verhaftet sind. Gleichwohl wird es „auch in Zukunft im räumlichen Gesamtgefüge der Bundesrepublik , Verliererregionen' geben, und nicht jede Region aus der Peripherie wird auf einen günstigen Wachstumspfad gelangen können“ (Akademie für Raumforschung und Landesplanung 2008: 10).

\section{Literatur}

Akademie für Raumforschung und Landesplanung (ARL) (2008): Politik für periphere, ländliche Räume: Für eine eigenständige und selbstverantwortliche Regionalentwicklung. Hannover. = Positionspapier aus der ARL, Nr. 77.

Beetz, S. (2006): Ländliche Politik im demographischen Wandel. In: Aus Politik und Zeitgeschichte 21-22, 25-32.

Beetz, S.; Huning, S.; Plieninger, T. (2008): Landscapes of Peripherization in North-Eastern Germany's Countryside: New Challenges for Planning Theory and Practice. In: International Planning Studies 13, 4, 295-310.

Bölsche, J. (2006): Verlassenes Land, verlorenes Land. Online unter: http://www.spiegel.de/politik/deutschland/0,1518,404888,00. html (letzter Zugriff am 04.03.2010).

Bucher, H.; Schlömer, C. (2008): Räumliche Varianz des demographischen Wandels. In: Geographie und Schule 172, 11-17.

Bundesamt für Bauwesen und Raumordnung (2000): Raumordnungsbericht 2000. Bonn. $=$ Berichte, Nr. 7.

Bundesamt für Bauwesen und Raumordnung (2005): Raumordnungsbericht 2005. Bonn. = Berichte, Nr. 21.

Diercke Weltatlas - Ausgabe 2008. Braunschweig.

Doering-Manteuffel, S. (1995): Die Eifel. Geschichte einer Landschaft. Frankfurt am Main, New York.

Glaser, R.; Gebhardt, H.; Schenk, W. (2007): Geographie Deutschlands. Darmstadt.

Hauss, F.; Land, R.; Willisch, A. (2006): Umbruch der Agrarverfassung und Zerfall der ländlichen Gesellschaft. In: Aus Politik und Zeitgeschichte 37, 31-38.

Heinrichs, B. (2006): Die neuen Leitbilder der Raumentwicklung - eine Neujustierung, aber kein Paradigmenwechsel. In: Informationen zur Raumentwicklung 11/12, 653-658.

Henkel, G. (1978): Die Entsiedlung ländlicher Räume Europas in der Gegenwart. Paderborn.
Hentschel, U. (2008): Aufmerksamer Blick in die Nachbarschaft. Online unter: http://www.lokalo.de/zeitung/3879/AufmerksamerBlick-in-die-Nachbarschaft (letzter Zugriff am 19.04.2008).

Herrenknecht, A. (1995): Der Riß durch die Dörfer - Innere Umbrüche in den Dörfern der neuen Bundesländer. In: Agrarsoziale Gesellschaft e. V. (Hrsg.): Dorf- und Regionalentwicklung in den neuen Bundesländern. Beiträge aus der Praxis. Göttingen, 50-64. = ASG-Kleine Reihe, Nr. 54

Hübler, K.-H.; Kujath, H. J. (2007): Ausgangslage und neue Herausforderung für die Raumentwicklungspolitik in Nordostdeutschland. In: Kujath, H. J.; Schmidt, S. (Hrsg.): Umbau von Städten und Regionen in Nordostdeutschland. Handlungsnotwendigkeiten und Handlungsperspektiven. Hannover, 9-28. = Arbeitsmaterial der ARL, Nr. 330

Keim, K.-D. (2006): Peripherisierung ländlicher Räume - Essay. In: Aus Politik und Zeitgeschichte 37, 3-7.

Keppler, D.; Land, R.; Laufer, D.; Nölting, B.; Ohlhorst, D.; Powalla, O.; Thomas, M.; Walk, H. (2009): Neue Energie im Osten. Die Energiewende als Chance für eine ökologische Reindustrialisierung Ostdeutschlands. Vorschläge des Innovationsverbunds Ostdeutschlandforschung. Online unter: http://www.ztg.tu-berlin. de/innovationsverbund-ostdeutschlandforschung/download/Thesen EE in Ostdeutschland.pdf (letzter Zugriff am 05.03.2010).

Klüter, $\bar{H}$. (2009): Stärken und Schwächen des Ländlichen Raumes in Mecklenburg-Vorpommern - unveröffentlichtes Manuskript.

Kocks, M. (2004): Infrastruktur und demographischer Wandel: Die Anpassung managen. In: LEADER Forum 2, 25-27.

Land, R.; Thie, H. (2009): Das Varchentiner Modell. In: Links, C.; Volke, K. (Hrsg.): Zukunft erfinden. Kreative Projekte in Ostdeutschland. Berlin, 115-122.

Land, R.; Willisch, A. (2006): Schrumpfung - Raumordnung oder Gesellschaftsordnung? In: Berliner Debatte Initial 17, 5, 54-64.

Maack, K.; Grundmann, M.; Kreft, J.; Lewandowska, A.; Voss, E. (2005): Wachstumspol Stettin und Auswirkungen auf die Entwicklung der deutsch-polnischen Grenzregion. Düsseldorf. = edition der Hans-Böckler-Stiftung, Nr. 132.

Peters, R.-H. (2007): Lasst die Wölfe rein! In: Stern 13, 184-191.

Reichert-Schick, A. (2008): Siedlungsregression und Schrumpfungsprozesse ländlicher Gemeinden in Vorpommern. In: Europa Regional 16, 1, 36-48.

Reißig, R. (2003): Das ganz andere Gefühl für Brüche könnte ein groBer Kulturvorteil sein. Neues Nachdenken über Ostdeutschland am Ende der Nachwendezeit: ein Auslauf- oder ein Zukunftsprojekt? In: Neues Deutschland vom 29.03.2003, 24.

Schwedt, H. (1984): Abwanderung und Dorfkultur. In: Schwedt, H.. (Hrsg.): Migration und Dorfkultur. Untersuchungen in Abwanderungsregionen des Landes Rheinland-Pfalz. Mainz, 3-68. = Mainzer Studien zur Sprach- und Volksforschung, Nr. 7.

Statistisches Amt Mecklenburg-Vorpommern (2007): MecklenburgVorpommern - Gemeindedaten 2007 (CD-Rom). Schwerin.

Statistisches Landesamt Rheinland-Pfalz (2009): Datenbank „Meine Heimat: mein Dorf, meine Stadt“. Online unter: http://www.infothek.statistik.rlp.de/lis/MeineRegion/index.asp (letzter Zugriff am 08.07.2009).

Stöber, S. (2006): Lebendige Dörfer in Brandenburg - Bürgerbeteiligung im Alltag. Ergebnisse einer Befragung in 5 Dörfern. Online unter: http://www.la21bb.de/service/stoeber_1.pdf(letzter Zugriff am 26.08.2009).

Weiß, W. (2006): Zur Entwicklung einer Residualbevölkerung infolge lang anhaltender selektiver Abwanderung in Mecklenburg-Vorpommern. Auswirkungen der Bevölkerungsalterung unter besonderer Berücksichtigung regionaler Aspekte. In: Zeitschrift für Bevölkerungswissenschaft 31, 3-4, 469-506.

Wichmann, S. (2007): Regionale Identität und Landschaftskult als Hemmnis der Abwanderung? In: Denkanstöße: Landschaftskult - Kulturlandschaft 6, 66-75. 